\title{
A few thoughts on diabetic striatopathy - case report and short review
}

\author{
Ioan-Cristian Lupescu ${ }^{1}$, Ioana-Gabriela Lupescu ${ }^{2,3}$, Anca Arbune ${ }^{1}$, \\ Basel Toron', Adriana Octaviana Dulamea ${ }^{1,4}$ \\ ${ }^{1}$ Neurology Department, Fundeni Clinical Institute, Bucharest, Romania \\ ${ }^{2}$ Radiology and Imaging Department, Fundeni Clinical Institute, Bucharest, Romania \\ ${ }^{3}$ Radiology and Imaging Department, \\ "Carol Davila" University of Medicine and Pharmacy, Bucharest, Romania \\ ${ }^{4}$ Neurology Department, "Carol Davila" University of Medicine and Pharmacy, Bucharest, Romania
}

\begin{abstract}
Diabetic striatopathy is an uncommon complication of diabetes mellitus, usually characterized by hemichorea-hemiballismus with T1-high signal intensities involving the contralateral striatum. We present the case of an 81 years-old woman with poorly controlled type 2 diabetes mellitus, who was rushed to the hospital for acute-onset right-sided hemiparesis, somnolence and aphasia. Blood analysis revealed hyperglycemia $(412 \mathrm{mg} / \mathrm{dl})$ and raised level of glycated hemoglobin (14.5\%), while head CT examination showed a spontaneous hyperdensity involving the left caudate and lenticular nuclei, with no edema. Brain MRI was also performed and highlighted T1-hyperintensities at the level of the same structures, with no diffusion restriction. The patient improved significantly under adjustment of insulin therapy. Based on these observations, a diagnosis of diabetic striatopathy was established. We present a short review of the literature regarding this condition and its main characteristics.
\end{abstract}

Keywords: diabetes mellitus, hyperglycemic encephalopathy, striatopathy, hemichoreahemiballismus, insulin therapy

\footnotetext{
Abbreviations (in alphabetical order)

CT - Computer Tomography

FDG - Fluorodeoxyglucose

FLAIR - Fluid attenuation inversion recovery

MRI - Magnetic Resonance Imaging

Naa - N-acetylaspartate

Pe T - Positron e mission Tomography

s WI - s usceptibility Weighted Imaging
}

\section{INTRODUCTION}

Hyperglycemic encephalopathy is a well-known complication of diabetes mellitus and can present either as hyperglycemia with ketoacidosis or as hyperosmolar nonketotic hyperglycemia [1]. The degree of insulin is what determines development of one or the other. Insulin is $\square$ near absolute in diabetic ketoacidosis, leading to marked catabolism of glycogen, triglycerides and amino acids, with consequent synthesis of ketone bodies, while in hyperosmolar hyperglycemia, there is enough insulin to prevent ketogenesis, but not for glucose utilization [2].

a set of diagnostic criteria has been proposed for the two by the a merican Diabetes a ssociation (see table 1) [3,4]. 
TABLE 1. Diagnostic criteria for diabetic ketoacidosis and hyperosmolar nonketotic hyperglycemia (4)

\begin{tabular}{|l|c|c|}
\hline Parameter & $\begin{array}{c}\text { Diabetic } \\
\text { ketoacidosis }\end{array}$ & $\begin{array}{c}\text { Hyperosmolar } \\
\text { nonketotic } \\
\text { hyperglycemia }\end{array}$ \\
\hline Blood glucose $(\mathrm{mg} / \mathrm{dl})$ & $>250$ & $>600$ \\
\hline $\begin{array}{l}\text { Effective serum } \\
\text { osmolality (mOsm/kg) }\end{array}$ & Variable & $>320$ \\
\hline Arterial pH & $\leq 7.30$ & $>7.30$ \\
\hline $\begin{array}{l}\text { Serum bicarbonate } \\
\text { (mEq/l) }\end{array}$ & $\leq 18$ & $>18$ \\
\hline Ketone bodies & $\begin{array}{c}\text { Positive in blood } \\
\text { and urine }\end{array}$ & Absent/small \\
\hline Anion gap & $>10$ & Variable \\
\hline
\end{tabular}

Under the term diabetic striatopathy, we describe a syndrome encountered usually in the hyperosmolar hyperglycemic state (and less often in diabetic ketoacidosis), manifested clinically by hemichorea-hemiballismus and radiologically by T1 hyperintensities in the contralateral basal ganglia [5]. Its recognition is important, since it is potentially life-threatening, but also reversible with correction of hyperglycemia [6].

\section{CASE PRESENTATION}

a n 81 years-old woman was brought in by the ambulance for sudden onset of right-sided motor and aphasia. Medical history revealed type 2 diabetes mellitus (complicated by neuropathy, retinopathy and grade IV peripheral artery disease with left thigh amputation), grade III hypertension, dyslipidemia, heart failure and silent coronary artery disease. The patient was receiving high doses of insulin therapy at home, both rapid-acting (24 units at breakfast, 24 units at lunch and 20 units at dinner) and slow-acting (24 units at breakfast and 52 units in the evening), as well as antiplatelet, lipid-lowering and antihypertensive medications.

The clinical picture was compatible with a stroke in the territory of the left middle cerebral artery, since the patient presented right-sided hemiparesis, right-sided Babinski sign, somnolence and was unable to speak or understand.

On admission, a series of abnormal blood parameters were highlighted, notably hyperglycemia $(412 \mathrm{mg} / \mathrm{dl})$, raised lactate $(5.5 \mathrm{mmol} / \mathrm{l})$ and raised glycated hemoglobin/Hba 1c (14.5\%) (see Table 2).

TABLE 2. Abnormal biomarkers found in our patient

\begin{tabular}{|l|c|c|}
\hline Biomarker & $\begin{array}{c}\text { Patient } \\
\text { value }\end{array}$ & $\begin{array}{c}\text { Normal } \\
\text { range }\end{array}$ \\
\hline Glycemia $(\mathrm{mg} / \mathrm{dl})$ & 412 & $74-106$ \\
\hline Uric acid $(\mathrm{mg} / \mathrm{dl})$ & 12.1 & $2.6-7.2$ \\
\hline Creatinine $(\mathrm{mg} / \mathrm{dl})$ & 1.86 & $0.7-1.3$ \\
\hline Urea $(\mathrm{mg} / \mathrm{dl})$ & 116 & $12-45$ \\
\hline C-reactive protein (mg/dl) & 78.4 & $0-3$ \\
\hline Fibrinogen (mg/dl) & 612 & $180-350$ \\
\hline Aspartate transaminase (U/l) & 143 & $0-34$ \\
\hline Alanine transaminase (U/l) & 80 & $0-49$ \\
\hline Total bilirubin (mg/dl) & 2.5 & $0.1-1.2$ \\
\hline Alkaline phosphatase (U/l) & 178 & $35-130$ \\
\hline Gamma-glutamyl transferase (U/l) & 318 & $0-55$ \\
\hline
\end{tabular}

Head CT was urgently performed and revealed a spontaneous hyperdensity (between 40 and 55 units) at the level of the left caudate and lenticular nuclei, without peripheral edema 1). Hemorrhage was excluded, since the lesion was well demarcated (and limited to the structures men-
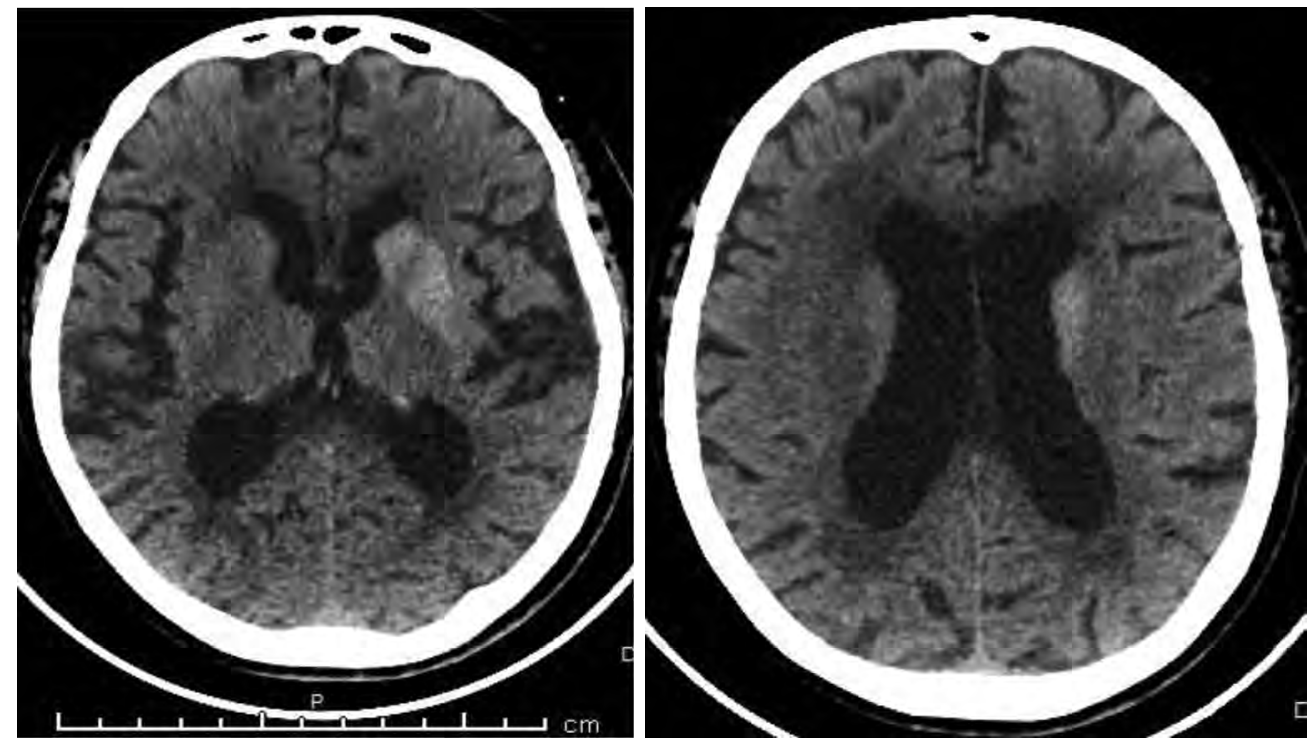

FIGURE 1. Non-enhanced head CT: Spontaneous hyperdensity involving the left caudate and lenticular nuclei 
tioned) and did not have a high enough density to be hemorrhage (which is between 60 and 80 units). As such, the hyperdensity was considered an abnormality secondary to the hyperglycemic state. Other changes consisted of diffuse white matter hypodensities in the frontal-parietal periventricular region (possibly due to small vessel disease) and hydrocephalus (Evans index of 0.37) without an obvious obstructive etiology.

a ssessment was completed with brain MRI, which showed T1-hyperintense and T2/FLa IR-hypointense lesions involving the left caudate and lenticular nuclei, without diffusion restriction. There were also hemosiderinic spots (s WI-hypointense) bilaterally at the level of the temporal lobes, as well as T2* hypointensities involving the basal
Insulin therapy was reassessed by an endocrinologist, with clinical improvement soon afterwards. a t discharge, the patient was conscious, with spontaneous and comprehensible speech and mild right-sided hemiparesis.

\section{DISCUSSIONS}

a rare condition, diabetic striatopathy (Ds ) was believed to be unique to Asians, however a study conducted by shafran et al. noted its existence in the Western population too. a nalyzing retrospectively 697 patients with elevated HbAlc $(>10 \%)$, they found 4 patients who presented with hemichorea/choreoathetosis and imaging compatible with Ds (only one of which was diagnosed as such during hospitalization) [7].
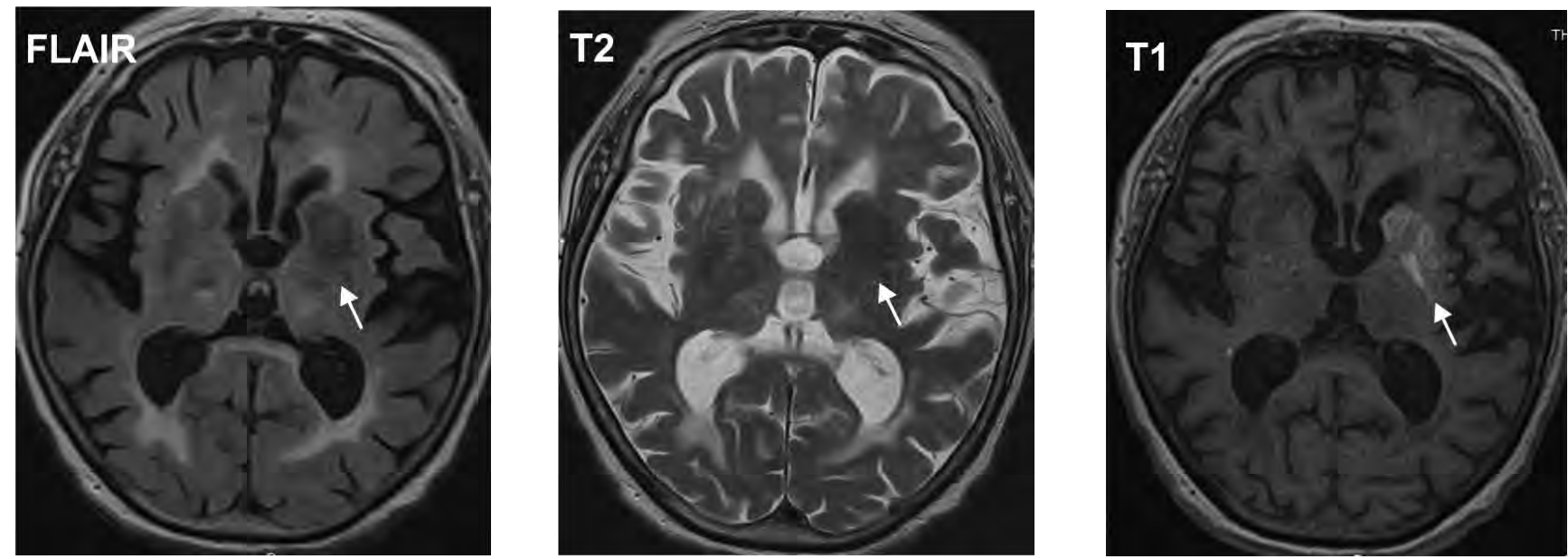

FIGURE 2. Brain MRI - T1-hyperintense and T2/FLAIR hypointense lesion at the level of the left caudate and lenticular nuclei (white arrow). Periventricular white matter hyperintensities visible on FLAIR (leukoaraiosis). Slight cerebral atrophy
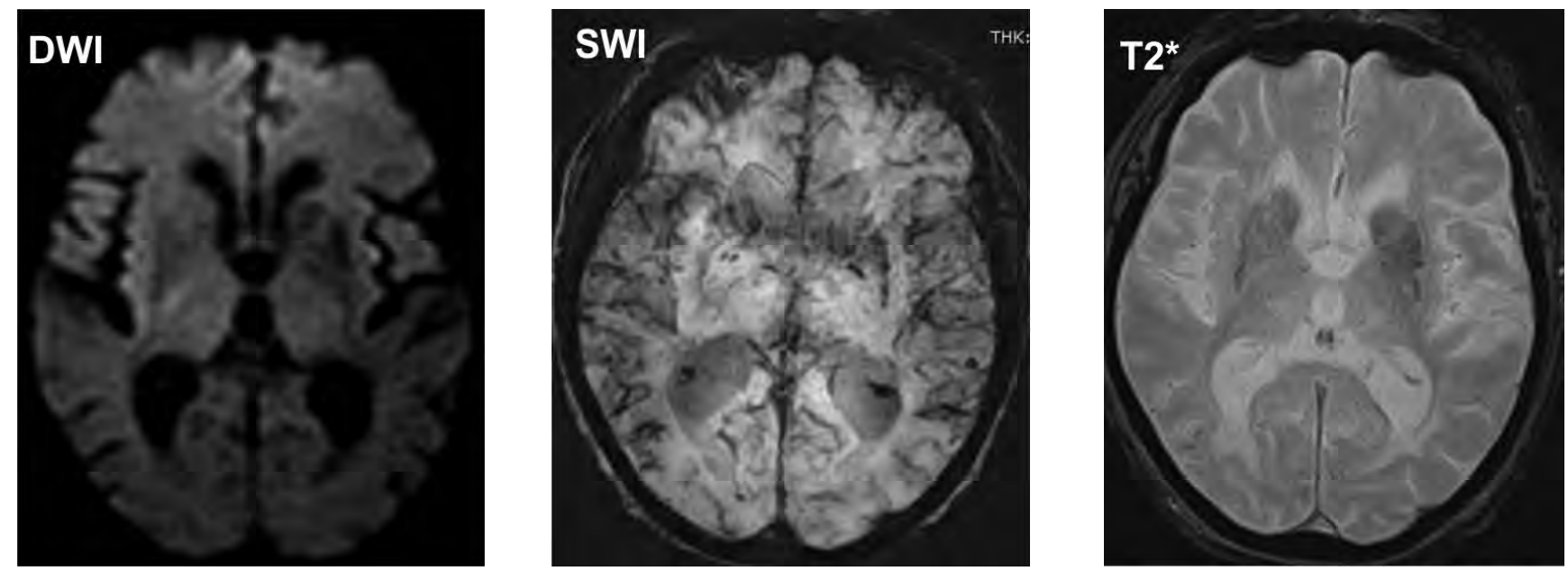

FIGURE 3. Brain MRI - The lesion shows no diffusion restriction on DWI 
By using MRI (in 6 patients), FDG-Pe T (in 1 patient) and biopsy of the striatum (in 1 patient), a be et al. highlighted the main imaging and pathological characteristics of Ds and attributed the condition to obliterative vasculopathy [8].

TABLE 3. Imaging and pathological findings in diabetic striatopathy (8)

\begin{tabular}{|l|l|}
\hline Method used & Findings \\
\hline \multirow{4}{*}{ MRI } & $\begin{array}{l}\text { T1-hyperintensity of the affected } \\
\text { striatum }\end{array}$ \\
\hline \multirow{5}{*}{ MR-Spectroscopy } & $\begin{array}{l}\text { Affected striatum: } \\
- \text { decreased NAA/creatine ratio } \\
- \text { normal choline/creatine ratio } \\
- \text { myoinositol peak }\end{array}$ \\
\cline { 2 - 3 } & $\begin{array}{l}\text { Contralateral striatum: } \\
- \text { decreased NAA/creatine ratio } \\
- \text { increased choline/creatine ratio } \\
- \text { no myoinositol peak }\end{array}$ \\
\hline \multirow{2}{*}{ FDG-PET } & $\begin{array}{l}\text { Decreased FDG accumulation in the } \\
\text { affected striatum }\end{array}$ \\
\hline Biopsy - & $\begin{array}{l}\text { Patchy necrosis, arteriolar wall } \\
\text { thickening with hyaline degeneration, } \\
\text { capillary proliferation, erythrocyte } \\
\text { extravasation and infiltration of } \\
\text { lymphocytes/macrophages }\end{array}$ \\
\hline
\end{tabular}

Ds affects primarily elderly females with hyperosmolar hyperglycemia, but cases have been described in other groups as well [9]. For example, Das et al. reported the occurence of Ds in two young males with diabetic ketoacidosis. There have been only a few cases of Ds so far in children, including one in a teenager with subacute onset of hemichorea accompanied by weight loss, polydipsia and polyuria [10].

Although DS develops in the setting of nonketotic hyperglycemia, its onset may be delayed by some weeks after the episode, even when the hyperglycemic state has been controlled [11]. Ds is considered reversible under insulin therapy, but if left untreated, Ds may determine persistent symp-

\section{REFERENCES}

1. Allan HR, Martin AS, Joshua PK, Sashank P. The Acquired Metabolic Disorders of the Nervous System. In: Allan HR, Martin AS, Joshua PK, Sashank P. Adams and Victor's Principles of Neurology - 11th Edition. McGraw-Hill Educational, 2019:1165-1166.

2. Gosmanov AR, Gosmanova EO, Kitabchi AE. Hyperglycemic Crises: Diabetic Ketoacidosis (DKA), And Hyperglycemic Hyperosmolar State (HHS) [Updated 2018 May 17]. In: Feingold KR, Anawalt B, Boyce A et al., editors. Endotext [Internet]. South Dartmouth (MA): MDText.com, Inc.; 2000.

3. Kitabchi AE, Umpierrez GE, Murphy MB, Kreisberg RA. Hyperglycemic Crises in Adult Patients with Diabetes. A consensus statement toms and irreversible structural lesions [12]. Lucassen et al. noted marked atrophy of the right caudate nucleus in a woman with poorly controlled type 2 diabetes and persistent choreic movements of the left upper limb (of 4 years duration). There is also the possibility of recurrence of Ds, as was shown by Lin et al. in a 69-years-old female who suffered a relapse of hemichorea-hemiballismus during a period of normoglycemia. There was a corresponding waxing-and-waning $\mathrm{T} 1$ change of the striatum on MRI [13].

Ds has been reported to occur without chorea and ballismus. $\mathrm{s}$ ato et al. described the case of a 58-years-old male with T1-hyperintense lesions of the striatum, but who presented only with severe altered consciousness [14]. Ds can represent the manifestation of diabetes mellitus and should be suspected in patients with sudden onset of hemichoreic movements without a prior history of diabetes [15].

a lthough usually unilateral, Udare et al. reported a case of bilateral DS in a 79-years-old male with known type 2 diabetes, who presented with bilateral chorea-ballismus and altered sensorium [16]. Notheworthy is also the case described by Lin et al., in which diabetic striatopathy coexisted with another rare diabetes-related complication, namely moyamoya disease [17].

\section{CONCLUSIONS}

Diabetic striatopathy is uncommon, but should be suspected in the appropriate setting of acute-onset movement disorder and hyperglycemia, in patients with or without prior history of diabetes mellitus. Our case was particular since the patient had no hemichorea-hemibalismus syndrome, but rather a clinical picture mimicking stroke.

Conflict of interest: none declared Financial support: none declared

from the American Diabetes Association. Diabetes Care. 2006; 29(12):2739-2748.

4. Kitabchi AE, Umpierrez GE, Miles JM, Fisher JN. Hyperglycemic crises in adult patients with diabetes. Diabetes Care. 2009; 32(7):1335-1343.

5. Ehrlich M. Diabetic Striatopathy with Isolated Sensorimotor Symptoms Masquerading as Intraparenchymal Hemorrhage (P1.038). Neurology. 2015; 84(14):P1.038.

6. Özgür $A$, Esen $K$, Kaleağası $\mathrm{H}$ et al. Diabetic striatopathy in a patient with hemiballism. Emerg Radiol. 2015;22(3):347-9. 
7. Shafran I, Greenberg G, Grossman E et al. Diabetic striatopathy Does it exist in non-Asian subjects? Eur J Intern Med. 2016; 35:51-54.

8. Abe $Y$, Yamamoto $T$, Soeda $T$ et al. Diabetic striatal disease: Clinical presentation, neuroimaging, and pathology. Intern Med. 2009; 48(13):1135-1141.

9. Das L, Pal R, Dutta P, Bhansali A. "Diabetic striatopathy" and ketoacidosis: Report of two cases and review of literature. Diabetes Res Clin Pract. 2017; 128:1-5.

10. Faundez T, Klee P, Hanquinet S et al. Diabetic Striatopathy in Childhood: A Case Report. Pediatrics. 2016;137(4).

11. Lin CJ, Huang P. Delayed onset diabetic striatopathy: Hemichoreahemiballism one month after a hyperglycemic episode. Am J Emerg Med. 2017; 35(7):1036.

12. Lucassen EB, Delfyett WT, Stahl MC. Persistent Hemichorea and Caudate Atrophy in Untreated Diabetic Striatopathy: A Case Report. Case Rep Neurol. 2017; 9(3):299-303.
13. Lin YT, Chen SC, Yip PK et al. Magnetic resonance imaging volumetric analysis for diabetic striatopathy with two episodes of hemichorea-hemiballism syndrome: A case report. Medicine (Baltimore). 2019; 98(38):e17249.

14. Sato $\mathrm{H}$, Hamano M, Fushimi $E$ et al. Diabetic striatopathy manifesting as severe consciousness disturbance with no involuntary movements. Diabet Med. 2017; 34(12):1795-1799.

15. Vasudevan V, Laway BA, Wani AI, Wani MM. Chorea Associated with Nonketotic Hyperglycemia (Diabetic Striatopathy) in an Elderly Male. Indian J Endocrinol Metab. 2018; 22(6):859-860.

16. Udare AS, Sankhe S, Mondel PK. Bilateral diabetic striatopathy. Asian J Neurosurg. 2016; 11(2):169.

17. Lin JB, Sng AA, Wang FS et al. Acute hemichorea in a young type 1 diabetic. Int J Neurosci. 2019:1-3. 